\title{
Oncology: histopathology and imaging in the future
}

\author{
Neil J. Sebire
}

(C) Springer-Verlag 2011

The established gold standard for many diagnoses, in particular the diagnosis of paediatric malignant disease, is histological examination of tissue samples following biopsy. This review will focus on recent developments regarding the use of new imaging techniques to imply histological tissue characteristics in order to optimise sampling approach and maximise clinical yield of diagnostic and prognostic information.

Histological diagnosis has changed dramatically in recent decades. Having been based for more than 100 years on light microscopic examination of morphological features following routine stains, in the 1970-1990s many malignant diagnoses were revolutionised by the routine introduction of immunohistochemical staining using targeted antibodies to specific antigens present in fixed material. Similarly, in the last decade, the widespread introduction of molecular diagnostic techniques such as fluorescence in-situ hybridisation (FISH) and polymerase chain reaction (PCR) has altered both the diagnostic approach and a requirement for tissue at biopsy in order to make definitive diagnoses.

In parallel with these changes, an increasing proportion of paediatric tumour diagnoses are now made on the basis of image-guided needle core biopsies rather than traditional open surgical biopsies, with associated reduced morbidity. Despite the fact that significantly less material is available for histological analysis using this approach, adequate material

Disclaimer Dr. Sebire has no financial interest, investigational or off-label uses to disclose.

\section{N. J. Sebire $(\square)$}

Great Ormond Street Hospital for Children,

Great Ormond Street,

London WC1N 3JH, UK

e-mail: nsebire@mac.com is obtained in $>95 \%$ of cases and, similarly, $>95 \%$ of diagnoses on needle core biopsy are correct, based on subsequent resections. There was initial pathologist reluctance to support the use of needle core biopsies due to worries that the sample may either be unrepresentative, too small for molecular studies or too small for morphological studies. However, with the introduction of widespread immunohistochemical staining and molecular techniques, the importance of traditional morphological-basic criteria has become reduced, whilst core biopsies provide more than adequate material for molecular-based diagnostic investigations, since only a tiny amount of tissue is required. However, optimising the use of such small samples requires specialist laboratories and expertise in tissue handling such that a panel of diagnostic investigations can be performed on limited material.

Compared to adult practice, paediatric tumour diagnosis is particularly fortunate in one respect, since the majority of malignancies are sarcomas rather than carcinomas, many of which have specific tumour-associated translocations that produce fusion gene products that are readily detectable by either FISH or PCR even on limited amounts of tissue. Since most of these translocations are believed to be pathogenic for the tumour, definitive diagnosis can be made on limited material. Furthermore, with the introduction of microarray profiling, the potential for vastly increased diagnostic, prognostic and biological information to be obtained from these minimally invasive samples is possible.

Nevertheless, the issue of representativeness and biopsying the optimal part of the tumour remains important. Recent data examining aspects of novel imaging techniques, in particular the apparent diffusion coefficient using diffusion-weighted MRI, seems to reliably identify areas of high cellularity in paediatric small round cell tumours, allowing both better determination of response to therapy and identification of 
areas of viable tumour for targeted biopsy. This, in combination with other functional imaging techniques such as PET scanning, allows targeting of biopsies with a high degree of accuracy. Studies examining correlation of PET positivity in paediatric tumours reveal good identification of residual viable tumour areas in both Wilms tumour and neuroblastic tumours (Fig. 1).

Despite these advances, whilst imaging techniques can provide more information now regarding tissue cellularity and viability, as described above, the nature of the tissue remains impossible to determine without histological diagnosis at biopsy, and false-positive PET scans have been reported, for example in areas of nonviable postchemotherapy change with florid fibroblastic reaction and macrophage infiltration.

A combination of ever-evolving imaging techniques in conjunction with rapid advances in laboratory diagnostic possibilities from more and more limited material is likely to mean that a combined approach between diagnostic radiology, interventional radiology and paediatric histopathology will allow more rapid and accurate diagnostic and prognostic information to be obtained from minimally invasive core biopsies for the vast majority of paediatric malignancies.

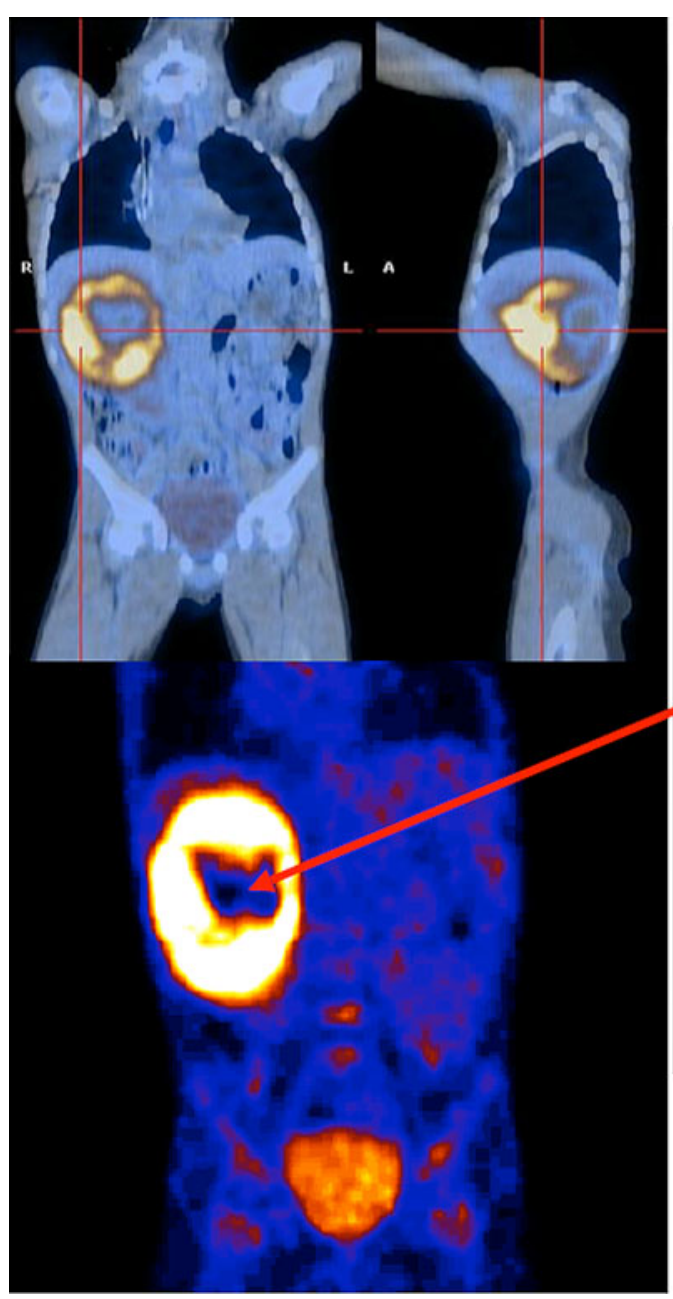

Fig. 1 Postchemotherapy Wilms tumour with ${ }^{18}$ F-fluoro-2deoxyglucose positron emission tomography (FDG-PET)-negative central necrosis. Surrounding the necrotic core, there is extensive FDG-PET-positive viable tumour, which showed diffuse anaplasia on histological examination. PET images (left), gross specimen (middle) with orientation corresponding to the coronal PET images, histological slides of necrotic (top right) and viable tumour tissue (bottom right) 